ARTÍCULO DE REVISIÓN

\title{
Complexus cordis
}

\section{Complexus cordis}

\author{
Hermes Ilarraza-Lomelí y María D. Rius-Suárez \\ Servicio de Rehabilitación Cardiaca y Medicina Física, Instituto Nacional de Cardiología Ignacio Chávez, Ciudad de México, México
}

\begin{abstract}
Resumen
El estudio científico del corazón nos ha permitido conocer su estructura y función profundamente, mediante la fragmentación y el análisis de sus partes, atendiendo a las pautas del método que tantos logros nos ha dado. Sin embargo, al momento de volver a ensamblar esos fragmentos analizados nos percatamos de que algo falta; simplemente la suma de las partes no hace al todo. Es así que, desde hace décadas, numerosos científicos han estudiado estrategias novedosas que permitan entender los fenómenos naturales desde modelos más incluyentes, abiertos e integradores, que atiendan con cercanía a las interacciones más que a los componentes. De esta manera, observamos que muchas variables suelen transgredir el plano convencional y parten hacia la no linealidad y la fractalidad, formando un tejido complejo que mantendrá su estructura mientras termodinámicamente sea viable. Así, en este documento se muestra la manera en que el estudio no lineal de la dinámica compleja cardiovascular comienza a darnos luz en muchas de las preguntas que a diario se plantea el cardiólogo clínico.
\end{abstract}

Palabras clave: Complejidad. Corazón. Entropía. Fractal. Infarto del miocardio. Insuficiencia cardiaca. No lineal.

\begin{abstract}
The science-based study of the heart has allowed us to know its structure and function deeply, through the fragmentation and analysis of its parts, following the guidelines that so many achievements have given to us. However, at the time of reassembling those analyzed fragments, we realize that something is missing; the simply sum of the parts is not equal to everything. Thus, for decades, numerous scientists have studied novel strategies that allow us understanding, every natural phenomena from a more inclusive, open and integrative models, which closely address to interactions rather than components. In this way, we can observe how, the behavior of many variables usually transgress the conventional plane and moves towards non-linearity and fractality, making a complex tissue that will maintain its structure while thermodynamically viable. Thus, this document shows the way how, the non-linear study of complex cardiovascular dynamics, begins to give us answers to many questions that the clinical cardiologist poses every day.
\end{abstract}

Key words: Complexity. Heart. Entropy. Fractal. Heart failure. Myocardial infarct. Non-linear.

«La medicina no escapa al signo de los tiempos; por ello, a partir del conocimiento de estos, es posible concebir a los organismos vivos como los sistemas complejos por excelencia, lo que obliga a realizar una revisión epistémica de los principios que la rigen." Javier Rosado ${ }^{1}$

\section{Correspondencia:}

*Hermes Ilarraza-Lomelí

E-mail: hermes_ilarraza@yahoo.com

1405-9940 / O 2020 Instituto Nacional CC BY-NC-ND (http://creativecommons.org/licenses/by-nc-nd/4.0/).

Disponible en internet: 24-11-2020

Arch Cardiol Mex. 2021;91(3):327-336
www.archivoscardiologia.com

Arch Cardiol Mex. 2021;91(3):327-336
www.archivoscardiologia.com

Fecha de recepción: 05-02-2020 Fecha de aceptación: 18-07-2020 DOI: 10.24875/ACM.200000391
}

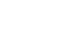

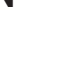


La naturaleza del ser humano lo ha conducido a la más profunda introspección para comprender esa misteriosa encrucijada entre su cuerpo y su espíritu: el corazón. En la actualidad, la ciencia declara concretamente que nuestro corazón es aquel «órgano de naturaleza muscular, común a todos los vertebrados y a muchos invertebrados, que actúa como impulsor de la sangre y que en el ser humano está situado en la cavidad torácica»². Sin embargo, el sentido común nos dice que, en realidad, estamos tratando con un fenómeno mucho más complejo, que podría estudiarse incluso desde disciplinas tan disímiles como la psicología, el esoterismo y la religión ${ }^{3}$.

La estructura y la función cardiovascular suele abordarse desde una perspectiva lineal y con modelos altamente controlados (cerrados), en la búsqueda de desarrollar ecuaciones que predigan su comportamiento. La estructura cardiaca se puede caracterizar desde lo macroscópico hasta lo subatómico, en un proceso de fragmentación y descripción que solemos denominar "análisis». Así, cada elemento de este órgano de bombeo es retirado e investigado a detalle de manera aislada, reduccionista. Una vez que el experto en el tema ha agotado sus elementos de observación y análisis, suele quedar convencido de conocer el fenómeno en profundidad. Ahora, solo queda rearmar todas las partes, amalgamando la información obtenida para aspirar a entender el funcionamiento del corazón en su totalidad, empresa que se convierte en un verdadero reto, dicho lo menos, interesante. Al terminar esta primera etapa, únicamente quedaría pendiente comprender, en un sentido ascendente, el papel del corazón dentro del cuerpo, la sociedad y el universo, ya que inevitablemente somos parte de un sistema abierto, en conexión permanente y obligada con nuestro entorno.

La estructura y la función del corazón se manifiestan principalmente como cambios en la dualidad materia-energía. Así, la luz que vemos, los sonidos que escuchamos, la presión o el calor que sentimos, son traducciones sensoriales de la naturaleza, que se expresa en formas tan caprichosas como el espectro electromagnético, el sonido o la termodinámica, entre otras. Es la tecnología la que nos ha permitido extender nuestra capacidad perceptiva más allá de nuestra fisiología y acceder a este lenguaje.

A través del conocimiento estructurado ofrecido por el método científico, el estudio del cuerpo humano alcanzó su máximo esplendor, y fueron personajes como Descartes y Newton, entre otros, los que entraron por primera vez en escena. Es aquí donde la asociación entre dos variables fue registrada en un plano cartesiano y analizada mediante geometría proceso matemático que da a la ciencia un carácter altamente predictivo, al tener fe en que la naturaleza tiene un comportamiento lineal.

En los últimos 500 años, la estructura macroscópica del ser humano ha sido descrita detalladamente por personajes como el polímata renacentista Leonardo da Vinci, seguido por un nutrido grupo de anatomistas como Andries van Wesel (De humani corporis fabrica) o Jean Léo Testut (Traité d'anatomie humaine). Con el advenimiento del microscopio (Zacharias Janssen, 1590), la descripción estructural se profundizó a un plano más elemental, pero no menos intrincado. La flamante «teoría celular» explicaría la manera en que los órganos y sistemas corporales están conformados, a su vez, por pequeños orgánulos (organelli), gracias a los estudios de científicos como Marcello Malpighi, Rudolf Virchow, Camillo Golgi y Santiago Ramón y Cajal. No obstante, el asunto no pararía ahí, ya que en los siglos XIX y XX personajes como John Dalton, Ernest Rutherford, Niels Bohr, Arnold Sommerfeld y Erwin Schrödinger desarrollaron un modelo que podría explicar la composición de la materia a niveles tan pequeños que escapaban a la platina del microscopio. Estas estructuras fueron reconocidas gracias a manifestaciones indirectas, es decir, a sus interacciones con otros elementos o fenómenos, y nació así el «modelo atómico", continente de un sinnúmero de estructuras. Al final, y en teoría, nuestro corazón podría «desarmarse» incluso en partículas subatómicas.

En una forma casi paralela, se ha estudiado la función cardiovascular. El papel del corazón como una bomba fue observado, probablemente, desde tiempos inmemoriales. En Europa, Galeno explicaba el proceso de salud y enfermedad a través de los pneumas (natural, vital y animal), y se creía que la sangre era mezclada en los ventrículos y «aireada» por una estructura llamada "traquearteria», para posteriormente ir al cerebro y, de ahí, a los nervios. Ya en pleno siglo versalites, William Harvey describiría la circulación sanguínea que conocemos hoy día (Exercitatio anatomica de motu cordis et sanguinis in animalibus), basándose en los trabajos de René Descartes y Michel Servet, entre otros.

Al otro lado del océano, la extracción del órgano vivo y latiendo, como parte de los rituales mexicas, derivó seguramente en su nombre náhuatl, Yóllotl, apelativo que significa corazón y círculo a la vez. Este comportamiento oscilatorio que inicia, tiene un desarrollo y vuelve al inicio es semejante al funcionamiento de un péndulo. Desde hace décadas, el estudio de la función 
cardiaca se ha basado en el llamado "ciclo cardiaco". Esta unidad funcional tiene múltiples expresiones determinadas por el método de observación, y van desde el «lub-dup» escuchado al acercar el oído al pecho de alguien hasta los más complejos métodos de análisis no lineal de la dinámica cardiaca ${ }^{4}$.

Uno de los grandes avances en la comprensión de la función cardiovascular es la agrupación simultánea de las diversas manifestaciones del ciclo cardiaco mediante el esquema de Wiggers-Lewis. Aquí se sobreponen, en la línea del tiempo, las gráficas obtenidas de los métodos tradicionales, como son el electrocardiograma (expresión electromagnética del intercambio iónico a través de la membrana del miocito), el fonomecanocardiograma (expresión acústica de las vibraciones secundarias al cierre y la apertura de las válvulas), el polígrafo hemodinámico (expresión de los cambios de la presión y del volumen sanguíneos dentro de las cámaras cardiacas) y la tensiometría arterial sistémica (expresión de los cambios de la tensión de los vasos sanguíneos en relación con la presión sanguínea y la onda viajera arterial). Cabe mencionar que todas estas gráficas son la «traducción visual» de los fenómenos físicos originales. Es así como podemos asociar los cambios eléctricos (excitación celular) con los cambios de volumen y presión intracavitarios (contracción celular) y el flujo sanguíneo (función valvular y dinámica de fluidos), en lo que pareciera un ciclo perpetuo y perfectamente periódico. La linealidad matemática de este esquema le confiere propiedades altamente predictivas, si nos apoyamos en la fisiología mecanicista de Descartes. La función de bomba del corazón pareciera culminar con la aparición del flujo sanguíneo, determinado por gradientes de presión, cambios de volumen y las propiedades intrínsecas de la sangre.

Por otro lado, desde un punto de vista termodinámico, la aparición de la bioquímica y de sofisticados métodos de estudio in vitro e in vivo nos ha mostrado que el organismo, y en particular el corazón, puede utilizar energía de manera casi inagotable, para poder contraerse vigorosamente un promedio de cien mil veces al día, con la menor cantidad de producción de calor, incluyendo la labor de otros órganos y sistemas como el digestivo y el endocrino.

Uno de los principios fundamentales de la teoría celular estriba en la homeostasis, concepto inicialmente descrito por Claude Bernard y refinado luego por Walter Cannon y Arturo Rosenblueth. En general, la homeostasis mantiene íntegras las estructuras y las funciones celulares, «más allá del equilibrio», mediante la generación de gradientes de diversa índole, a partir de complejos fenómenos adaptativos, muchos de ellos basados en mecanismos de realimentación. Así, el sistema busca mantener este desequilibrio, al menos durante un tiempo al que llamamos «existencia» (De Pomposo) $)^{5}$.

Los avances tecnológicos y la aparente sencillez de la función cardiaca de bombeo han entusiasmado a diferentes grupos de investigadores para desarrollar un dispositivo que sustituya por completo el órgano vital: el corazón artificial. Sin embargo, es aquí donde comienzan los problemas.

El marcapasos, por ejemplo, ha tenido un enorme éxito desde la mitad del siglo $x x$, cuando comenzó a mantener con vida a pacientes con ritmos muy lentos (disfunción del nodo sinusal o bloqueo auriculoventricular completo). En la actualidad se implantan miles de dispositivos diariamente y en el mercado podemos encontrar una gran diversidad de modelos con distintos programas y terapias, incluso con sofisticados protocolos de desfibrilación.

Por otro lado, en un intento por suplir la función de bomba del corazón, John Gibbon diseñó el primer sistema de soporte mecánico cardiovascular, conocido entonces como «dispositivo corazón-pulmón», que podía mantener un paciente vivo durante un par de horas, suficientes para realizar una cirugía intracardiaca. Poco tiempo después, esta bomba de circulación extracorpórea fue optimizada y se pudo incrementar su funcionamiento de horas a días ${ }^{6}$. El primer implante de un corazón artificial total lo realizó Denton Cooley (1969), mismo que mantendría con vida al paciente durante 64 horas antes de ser llevado a una cirugía de trasplante cardiaco. En la actualidad, los modernos dispositivos, como el SynCardia o el Soft Total Artificial Heart del ETH en Zúrich (Fig. 1), cuentan con cámaras cardiacas armadas con una bomba de flujo pulsátil, cuatro válvulas de disco y un generador neumático externo. El récord de funcionamiento de este tipo de dispositivos es de 1374 días, y la supervivencia de los pacientes durante el primer año es del $60 \%$. El corazón artificial total se ha utilizado en pacientes con rechazo de un injerto cardiaco, arritmias intratables, cardiopatías congénitas complejas o rotura ventricular. Este tipo de dispositivo sigue encaminado a cumplir el rol de «puente» entre el choque cardiogénico y el trasplante cardiaco, con una tasa de éxito que oscila entre el $60 \%$ y el $80 \%$.

Todo iba relativamente bien, hasta que comenzamos a observar que estas soluciones de alta tecnología no son tan efectivas como habíamos predicho. En relación con los marcapasos, podemos ver que todavía quedan 


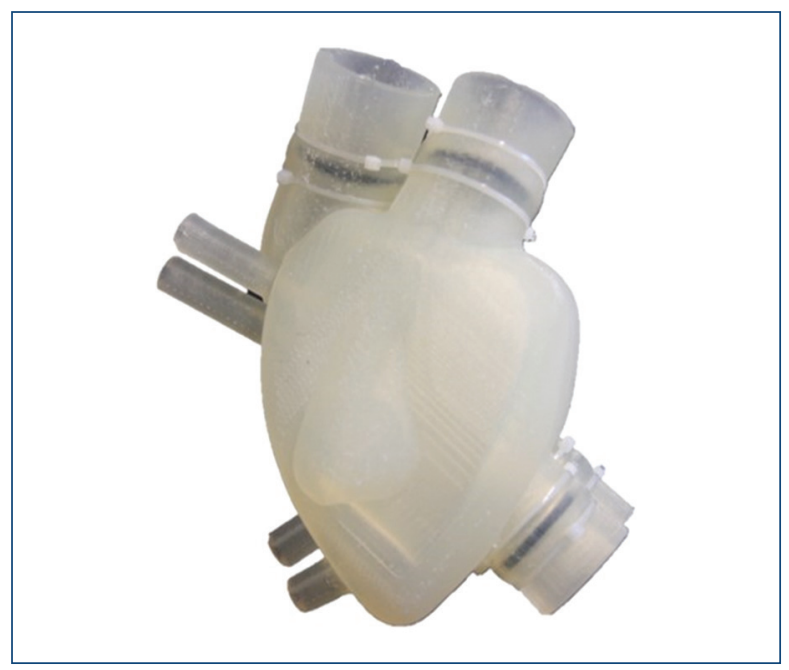

Figura 1. El corazón artificial total. Desde los años 1960 se han diseñado diversos modelos de corazón artificial, con el objetivo de poder sustituir la función de bombeo cardiaco, lo que se ha logrado por tiempo restringido y con resultados variables. Este es un modelo de corazón artificial "suave», desarrollado en Zúrich, que tiene la característica de parecerse más al corazón humano natural que sus predecesores.

problemas por resolver, como son la limitada vida de la batería, la exteriorización del generador, la infección del sitio de colocación, la colonización o la fractura de los electrodos, la interferencia electromagnética, la remodelación miocárdica patológica secundaria a la estimulación artificial y, sobre todo, la estimulación «rígida» y descoordinada con la vida real del paciente, a pesar del desarrollo de sofisticados sensores de actividad corpo$\mathrm{ral}^{7}$. Por otro lado, a pesar de las innovaciones hechas al corazón artificial, innumerables complicaciones atribuidas a su implante y al mantenimiento a mediano y largo plazo, y la falta de reintegración de los pacientes a una vida «normal», impiden que sea considerado como una terapia destino en estos casos ${ }^{8,9}$.

¿Por qué un corazón artificial no ha podido sustituir al órgano nativo si su fisiología depende simplemente de un par de variables y su comportamiento es predeciblemente cíclico? ¿Qué estamos pasando por alto? ¿Es el fundamento aristotélico de causa-efecto una estrategia adecuada para explicar el fenómeno cardiovascular?

De manera análoga, el clima es un fenómeno con características similares, determinado por pocas variables de comportamiento cíclico. Una intensa nevada o un clima cálido dependen de dos situaciones: la temperatura del aire y la altitud donde se hizo la medición. Así, las diferentes combinaciones de aire frío arriba y caliente

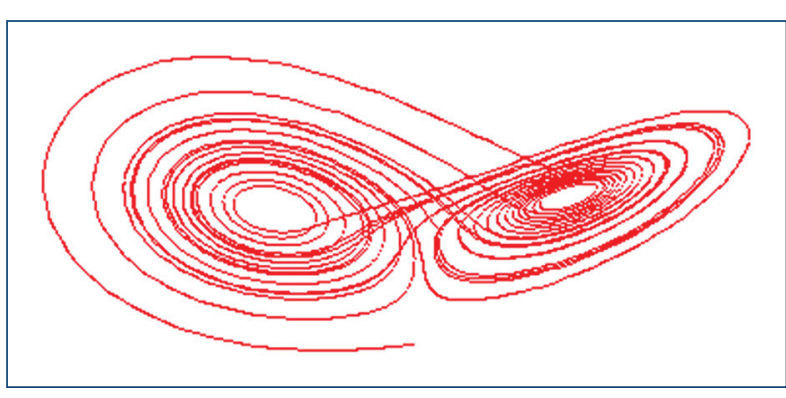

Figura 2. Atractor de Lorenz. En esta gráfica podemos observar algo muy semejante a lo que Lorenz consiguió en la búsqueda de un modelo lineal que le permitiera predecir los cambios climáticos.

abajo, o viceversa, generarán cambios atmosféricos específicos. Edward Norton Lorenz, matemático y meteorólogo, desarrolló una ecuación con la que podría predecir el clima con precisión, confiado en que se trataba de un fenómeno simple y teóricamente lineal. Así, observó que, si corría dos veces el mismo modelo matemático en su rudimentario equipo de cómputo, la aparición de mínimos cambios en las condiciones de inicio se asociaban a un resultado final drásticamente diferente $y$, por ende, impredecible. Sin embargo, al analizar los datos cuidadosamente, aunque no formaban una perfecta línea recta, sí generaron un gráfico con cierta morfología, reconocido como el atractor de Lorenz (Fig. 2), poco predecible, pero para nada aleatorio.

Por otro lado, el matemático Mitchell Jay Feigenbaum utilizó una ecuación logística, conocida como función de Feigenbaum, en la cual se asignó inicialmente un valor a $x$ y el número resultante lo asignaría nuevamente a $x$ al correr otra vez la fórmula:

$$
g(x)=-g\left(g\left(\frac{1}{\propto} x\right)\right)
$$

De esta manera, la ecuación se realimentaría continuamente y la gráfica final sería una serie de puntos originados por ella misma. Al principio se generó una línea que, posteriormente, presentaría una «bifurcación", y al seguir corriendo la gráfica se presentarían sobre ella más bifurcaciones, hasta un punto en que se observa un espectacular patrón que nadie podría haber predicho (Fig. 3). Además, al volver a correr la ecuación, el resultado final es el mismo. Así, algo que debería comportarse como una línea perfectamente predecible genera una gráfica completamente diferente, pero con cierta estructura. De algún modo, esta gráfica es producto de propiedades emergentes que van apareciendo y que desconocíamos. 


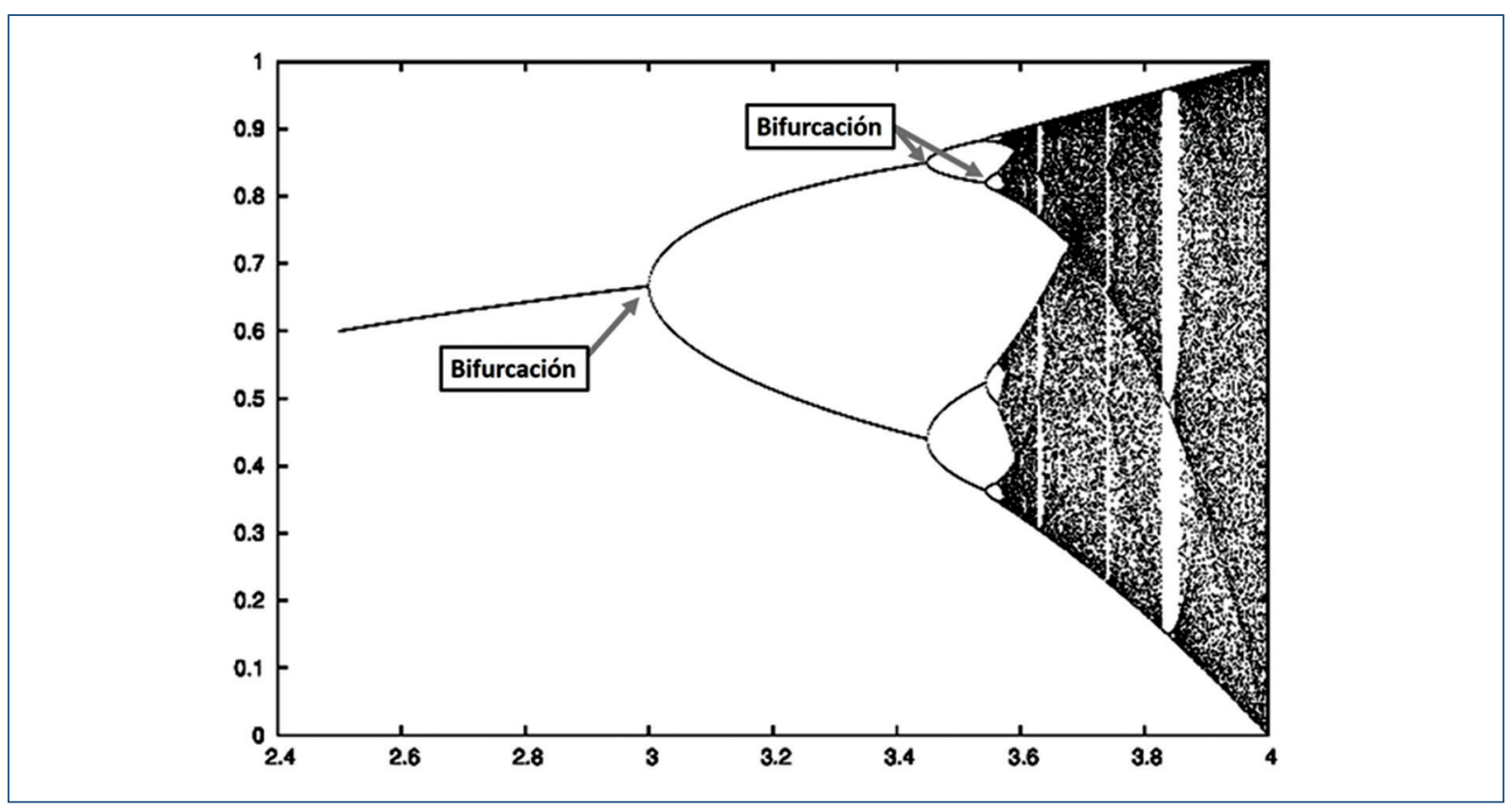

Figura 3. Diagrama de bifurcación de Feigenbaum. Esta gráfica muestra cómo una ecuación matemática, de manera independiente, puede tener propiedades emergentes como son las bifurcaciones, la fractalidad y en ocasiones zonas de caos, periodicidad y ruido ${ }^{49}$.

Si esto sucede con ciencias tan duras como la física o incluso el lenguaje matemático, qué podemos esperar del comportamiento del sistema cardiovascular. Así, la primera lección aprendida es que, incluso con pocas variables, un fenómeno puede tener un comportamiento «no lineal».

Edgar Morin explica que las variables existentes en la naturaleza se entretejen formando una intrincada red de conexiones. En este verdadero plexo de interacciones, si cada variable fuera un hilo, al tirar de uno habría una serie de reacciones locales y a distancia, producto de las interacciones de este con otros hilos, de los otros hilos con otros más y muchos de ellos entre sí. Esta estructura tisular facilita que nuevas propiedades emerjan del sistema, en ocasiones con leyes de comportamiento propias, como puede ser el fenómeno de resonancia o de autoorganización. Complexus significa lo que está tejido junto; en efecto, hay «complejidad cuando son inseparables los elementos diferentes que constituyen un todo y que existe un tejido interdependiente, interactivo e interretroactivo entre el objeto de conocimiento y su contexto, las partes y el todo, el todo y las partes, las partes entre ellas. Por esto, la complejidad es la unión entre la unidad y la multiplicidad» ${ }^{10}$. Así, lo "complejo» nace de la serie de interacciones de variables entretejidas, a diferencia de lo "complicado", que tiene su origen en nuestra incapacidad para comprender la naturaleza de un fenómeno (De Pomposo).

Por lo anterior, es casi inevitable sentir que la complejidad nos rodea, formando parte de innumerables sistemas que coexisten de una manera abierta, donde su particular dinámica no lineal va del comportamiento periódico al cuasi-periódico y al caos; la mayor parte del tiempo manteniendo su estructura contra la acción de las fluctuaciones o perturbaciones y, sobre todo, resistiendo la entrópica disipación que inevitablemente la llevara a caer en el equilibrio termodinámico final. A través del análisis de los sistemas complejos podremos observar las múltiples propiedades emergentes, donde el todo es más que la suma de sus partes; los atractores y la autoorganización que de ella emanan.

\section{Anatomía cardiovascular}

Una de las características morfológicas del sistema cardiovascular que más ha sido estudiada desde un punto de vista de la complejidad es el árbol vascular. La distribución espacial de los vasos sanguíneos posee una geometría interesante, ya que al igual que otras estructuras ramificadas tiene una dimensión fracciona$\mathrm{da}$, es decir, no bidimensional (2.0), pero tampoco enteramente tridimensional (3.0). La forma del árbol vascular 


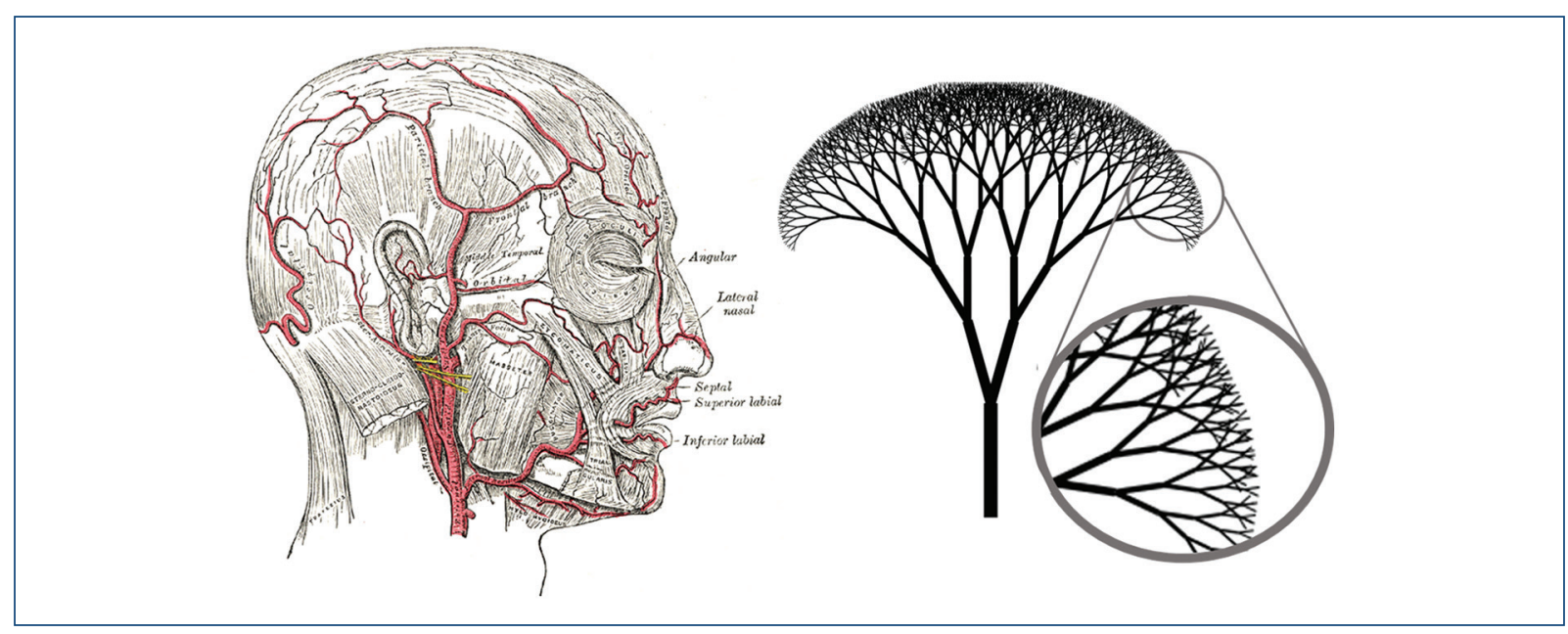

Figura 4. La fractalidad del árbol vascular. En este esquema se observa la característica dimensional del árbol arterial, misma que varía dependiendo del tamaño de los vasos, siempre con un componente fraccional. En la imagen de la derecha se aprecia que la distribución de los vasos es muy semejante, sin importar la escala.

es independiente de escala, siendo en los grandes vasos muy semejante a la de los vasos de menor calibre, y esta distribución sigue la ley de potencias. "Un fractal es una forma geométrica áspera o fragmentada que se puede dividir en partes, cada una de las cuales es una copia (al menos aproximadamente) de la reducción de tamaño de la totalidad $»^{11}$.La ramificación del árbol vascular le confiere diferentes niveles de organización, lo cual afecta las características del flujo sanguíneo (Fig. 4). Se ha calculado que el árbol arterial tiene una dimensión fractal promedio de 2.7, semejante a la del brócoli o la cromatina. La dimensión fractal varía desde 2.33 en los grandes vasos, donde el flujo es más turbulento, hasta muy cercano a 3 en los capilares ${ }^{11-13}$. Existen varios métodos para medir la fractalidad de una estructura, pero la dimensión fractal (Df) es la más precisa $^{14}$. Los conocimientos sobre fractalidad son de gran utilidad para los investigadores que se encuentran diseñando vasos sanguíneos artificiales, para la detección de insuficiencia cardiaca o de fibrilación auricular y la evaluación de la angiogénesis ${ }^{15,16}$.

\section{Ritmo cardiaco}

Uno de los fenómenos que con mayor interés se han estudiado es el comportamiento de la frecuencia cardiaca, mismo que tiene un comportamiento no lineal y también complejo. El inicio del ciclo cardiaco puede marcarse con la activación del nodo sinusal, evento determinado por la interacción de variables como la pendiente de despolarización diastólica espontánea, el flujo del sistema nervioso autónomo (simpático-parasimpático), la presión de la sangre sobre la pared de la aurícula, la temperatura corporal, etc.

El electrocardiograma puede brindarnos información importante acerca de la dinámica del ritmo cardiaco y la influencia del sistema nervioso autónomo, lo que conocemos como variabilidad de la frecuencia cardiaca, la cual muestra diversos patrones en el dominio del tiempo, de la frecuencia o de ambos, al ser estudiada con métodos de análisis no lineal. Entre ellos tenemos el análisis de reconstrucción del espacio de fase, el exponente de Lyapunov, la dimensión de correlación, análisis de fluctuaciones sin tendencia, el mapa de recurrencia, el mapa de Poincaré, la entropía aproximada y la entropía muestral, entre otros ${ }^{17,18}$.

Por un lado, Baillie et al..$^{19}$ declaran que la dinámica de la frecuencia cardiaca de un corazón sano es no lineal, no presenta caos de baja dimensión y es multifractal, lo que hace que el ritmo cardiaco no regrese al mismo estado de equilibrio estacionario después de ser afectado por alguna perturbación. En contraparte, estudios más recientes sostienen que el ritmo cardiaco tiene un comportamiento caótico en estrecha relación con el control del sistema nervioso autónomo ${ }^{20}$.

Diversos estudios muestran que el ciclo cardiaco en condiciones fisiológicas no es un oscilador perfectamente periódico, con dimensiones de correlación $\left(D_{2}\right)$ que van de 3.6 a $5.2^{21}$. El origen de un latido puede depender del latido previo o de la interacción de latidos precedentes, adoptando un comportamiento periódico, cuasi-periódico, caótico o francamente azaroso (ruido) ${ }^{22}$. 
Al hablar sobre la conducción y la propagación del estímulo, debemos recordar que el miocardio es un grupo de células con propiedades excitables y conductivas que conforman un sincicio, interconectado por uniones celulares de alta resistencia mecánica y baja resistencia eléctrica. Sin embargo, la despolarización de una célula podrá ser transmitida o no a las células vecinas, siempre y cuando se den las condiciones favorables para su propagación; fenómeno semejante a la autoorganización de los cardúmenes o las parvadas, y análogo a la resonancia mecánica, donde un cuerpo comienza a vibrar al ser sometido a la acción de una fuerza oscilatoria que tiene el mismo periodo de vibración de ese cuerpo $^{23}$. Por otro lado, la energía con la que se transmite el impulso eléctrico y mecánico en el miocardio alcanzará el resto del tejido si la magnitud es apropiada y, sobre todo, si la entropía termodinámica es adecuada. La entropía es una magnitud que mide, de alguna manera, el grado de organización de un sistema en el cual, a mayor azar, mayor entropía. Esta es una función de estado, de carácter extensivo que crece de manera natural, describiendo la «irreversibilidad termodinámica del sistema». El sistema cardiovascular es termodinámicamente disipativo y maneja una gran cantidad de información, aunque en general presenta una dinámica con baja entropía. La dinámica cardiaca estudiada mediante las «proporciones de entropía” puede ayudar al médico a identificar diversos tipos de enfermedades, en particular al evaluar fenómenos de "autoorganización», característica de los fenómenos complejos que reviste importancia en la regulación del sistema cardiovascular, desde la activación eléctrica del corazón hasta el control de la presión arterial.

La propagación espaciotemporal de la actividad eléctrica y contráctil del miocardio sigue diversos patrones; se observan frentes de onda planos, oscilatorios, circulares, espirales e incluso elipsoidales. Estos patrones están estrechamente relacionados con diversas variables, como la geometría y la disposición espacial de las fibras miocárdicas, la disposición de las subestructuras del miocito, y las concentraciones de $\mathrm{Ca}^{++}$ intracelular y de acetilcolina, entre otras. Estos patrones de propagación se observan incluso en los miocitos aislados, donde los diferentes orgánulos celulares pueden generar verdaderas microrreentradas ${ }^{24,25}$.

Debido a lo problemático de realizar estudios de conducción miocárdica in vivo, se han desarrollado modelos in silico, como el de Ten Tusscher-Noble-Noble-Panfilov (TNNP), que permite reproducir las condiciones iniciales excitables del miocardio y ver el efecto en la

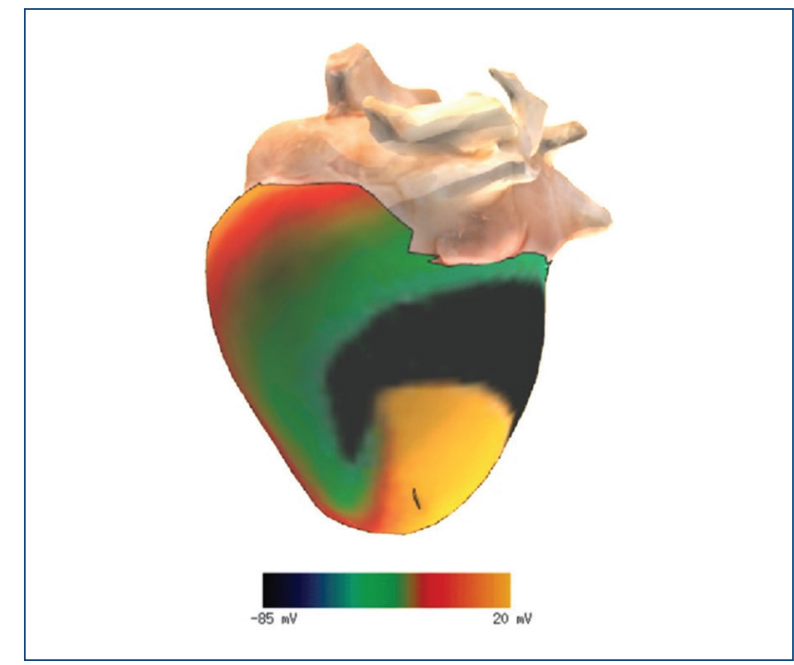

Figura 5. En este modelo in silico, presentado por Cherry y Fenton ${ }^{27}$, el internauta puede cambiar las características de las concentraciones de electrolitos o enviar una serie de extraestímulos, que generarán diversos tipos de frentes de onda de propagación en el miocardio virtual. Este en particular obedece a la clásica espiral observada durante una taquicardia ventricular.

propagación del frente de onda, inclusive en un plano tridimensional (Fig. 5) ${ }^{26}$. Entre otros hallazgos, se ha visto que la taquicardia ventricular está asociada con la formación de un frente de onda espiral que se propaga por el miocardio, mientras que en la fibrilación ventricular estos patrones tienen un comportamiento mucho más turbulento, asociados a la heterogeneidad en la forma de la fibra muscular y las concentraciones de los iones ${ }^{27-31}$.

La dinámica de la variabilidad de la frecuencia cardiaca también nos muestra que el sistema cardiovascular, además de ser complejo, es abierto. Así, diversos investigadores han demostrado que la inducción de estrés o de sobrecarga mental puede cambiar la dinámica no lineal de la frecuencia cardiaca ${ }^{32,33}$.

\section{Contracción muscular y hemodinámica}

La contracción cardiaca es un fenómeno mecánico que tiene, por un lado, una cadena jerárquica de eventos que se inician con la despolarización diastólica del nodo sinusal, el cual dispara los cambios en la concentración de iones, sobre todo del $\mathrm{Ca}^{++}$, que a su vez despolariza la célula, las células vecinas y el corazón entero. Sin embargo, por otro lado, diversos estudios han demostrado que las proteínas contráctiles (actina y miosina) no solo obedecen al nodo sinusal, sino que tienen 
propiedades oscilatorias autónomas, convirtiéndose en un verdadero «motor molecular» ${ }^{34}$.

Tradicionalmente, el flujo sanguíneo se ha estudiado desde la dinámica de fluidos newtoniana, mediante modelos de flujo estable como la ecuación de Poiseuille, la ecuación de Bernoulli, la ecuación de Casson y el número de Reynolds; estos involucran el comportamiento de las estructuras sanguíneas y vasculares. No obstante, debemos recordar que la sangre no es un simple líquido, sino un tejido; está compuesta por agua, electrolitos, células y otros elementos, cada uno con sus características y su comportamiento individual ${ }^{35}$. Además, el flujo sanguíneo no es uniforme a lo largo del sistema cardiovascular, pues muestra importantes diferencias en cuanto al tamaño, la forma y la disposición de los vasos y la composición de la sangre. Particularmente, en los vasos de pequeño calibre, como las arteriolas y los capilares, el tamaño de los elementos formes de la sangre, los cambios en la viscosidad y la baja tasa de cizallamiento parietal hacen que se comporte como un fluido no newtoniano. Por otro lado, el corazón, las válvulas cardiacas y los vasos sanguíneos tampoco son un mero sistema de bomba y tuberías; el árbol vascular y las estructuras cardiacas tienen una estructura fractal que determina claramente las características del flujo ${ }^{36}$. Además, la hemodinámica está determinada en parte por la tensión parietal y el estrés de cizallamiento, propiedades que tienen un comportamiento no lineal (ecuación de Casson) que puede afectar la integridad de las células hemáticas. Existen ya modelos no lineales para evaluar el grado de hemólisis asociado al contacto con la sangre de los dispositivos médicos ${ }^{37}$.

Por otro lado, la resistencia que ofrece la superficie del vaso o de una cámara cardiaca determina el grado de desplazamiento de la sangre; si la resistencia fuera nula, el flujo no existiría. El comportamiento del flujo sanguíneo en la circulación de pequeño calibre puede ser estudiada con mayor precisión a través del modelo de «dinámica de partículas disipativas» ${ }^{38}$.

Al estudiar el flujo sanguíneo debemos reparar en otras características que suelen ser excluidas, como su comportamiento oscilatorio, la transmisión a gran velocidad de la contracción ventricular por las paredes vasculares (onda viajera), la distribución fractal del árbol vascular o la interfase entre la columna sanguínea y el movimiento pulsátil de la pared del vaso. Para tal efecto se han desarrollado nuevos modelos, como la teoría del «receptor de aire» (windkessel), las ecuaciones de Wommersley y de Bessel, o el modelo de «dinámica molecular de granulación gruesa» de Karniadakis, que pretende incluir también el comportamiento de partículas subatómicas ${ }^{39,40}$.

Así, el ritmo cardiovascular es la resultante de la interacción de varios osciladores observados en el pulso cardiaco, la onda viajera, el flujo sanguíneo, el flujo del sistema nervioso autónomo e incluso osciladores iónicos intracelulares. Estos osciladores pueden tener un comportamiento periódico, cuasi-periódico, caótico o ruidoso, situación que favorece la fisiología o el estado patológico, según sea el caso ${ }^{41-43}$.

Según el esquema de Wiggers-Lewis, el flujo sanguíneo está estrechamente relacionado con diversas variables, como la apertura y el cierre de las válvulas cardiacas. Zheng y Guo ${ }^{44}$ propusieron un método de análisis no lineal del comportamiento de los ruidos cardiacos como un medio eficaz para la identificación de insuficiencia cardiaca crónica.

\section{Acoplamiento cardiopulmonar}

La ventilación y la circulación son dos procesos muy relacionados, y tanto la frecuencia cardiaca como la frecuencia respiratoria se encuentran acopladas fisiológicamente, lo cual se ha demostrado mediante el análisis convencional de la variabilidad de la frecuencia cardiaca y mediante el espectro de potencias en el dominio de la frecuencia ${ }^{45,46}$. Los estudios realizados en individuos bajo asistencia mecánica ventilatoria han mostrado que, al variar el modo de ventilación de asisto-controlado a ventilación espontánea con soporte de presión o sin soporte, se observan cambios en el comportamiento de la frecuencia cardiaca que van desde patrones lineales hasta una dinámica compleja, no lineal, que tiende a ser de tipo caótico. Este comportamiento se ha hecho evidente con el estudio de la complejidad de la dinámica no lineal, mediante análisis especializados del espacio de fase, que incluyen el exponente de Lyapunov, la entropía aproximada, la dimensión fractal, la dimensión de correlación y el exponente de Hurst, entre otros ${ }^{47}$. A la par, el análisis no lineal del acoplamiento cardiopulmonar permite identificar patologías como el síndrome de apnea-hipopnea obstructiva del sueño ${ }^{48}$.

El fenómeno de aterotrombosis, que lleva a la oclusión vascular y a la lesión del tejido diana (infarto tisular), pareciera tener una distribución regida por la ley de potencias, donde innumerables trombos muy pequeños son disueltos por el sistema fibrinolítico mientras que solo muy pocos trombos alcanzan el tamaño suficiente para causar la catástrofe vascular. Esta presentación es 
semejante a la observada con los movimientos telúricos ${ }^{49}$.

\section{Conclusión}

En resumen, a lo largo de su existencia el ser humano ha utilizado diferentes estrategias para entender su entorno, siendo el método científico el más utilizado en la actualidad, usualmente reduccionista y lineal; sin embargo, es evidente que, aun así, muchos aspectos fenomenológicos escapan a nuestra vista. Día a día, un creciente grupo de investigadores profundiza en este relativamente novedoso enfoque de estudio, en el que se aprecia a la naturaleza como un sistema complejo, abierto y no lineal; la perspectiva es otra, y el lenguaje y sus métodos también lo son. Acceder a este nuevo mundo no es sencillo, pero la recompensa bien vale la pena.

\section{Agradecimientos}

A Javier Rosado, Alexandre de Pomposo, Ady Castillo, Lilia Rodríguez, Aquiles Ilarraza, Rafael Chávez Domínguez y todos los que estudian la complejidad en nuestro país.

\section{Conflicto de intereses}

Ninguno.

\section{Financiamiento}

Ninguno.

\section{Responsabilidades éticas}

Protección de personas y animales. Los autores declaran que para esta investigación no se han realizado experimentos en seres humanos $\mathrm{ni}$ en animales.

Confidencialidad de los datos. Los autores declaran que han seguido los protocolos de su centro de trabajo sobre la publicación de datos de pacientes.

Derecho a la privacidad y consentimiento informado. Los autores declaran que en este artículo no aparecen datos de pacientes.

\section{Bibliografía}

1. Ruelas E, Mansilla R. Las ciencias de la complejidad y la innovación médica. Ensayos y modelos. México: Secretaría de Salud e Instituto de Física del Centro de Investigaciones Interdisciplinarias de Ciencias y Humanidades, UNAM; 2006
2. Diccionario de la Real Academia Española de la Lengua. Corazón. (Consultado el 15 de febrero de 2020.) Disponible en: https://dle.rae.es/ coraz $\%$ C3\%B3n?m=form

3. Ruelas-Barajas E, Mansilla-Corona R. Estado del arte de la medicina 2013-2014: las ciencias de la complejidad y la innovación médica: aplicaciones. Ciudad de México: Academia Nacional de Medicina, Editorial Intersistemas S.A. de C.V.; 2015.

4. Ilarraza $H$, Rius MD, Chávez R, Rodríguez L, llarraza A. Complexus cordis: salud y patología cardiovascular. En: Villegas M, Caballero L, Vizcaya E, editores. Biocomplejidad: facetas y tendencias. Ciudad de México: Coplt-arXives, Publishing Open Access with an Open Mind; 2019.

5. Ilarraza Lomelí H, Rius-Suárez MD. Arturo Rosenblueth Stearns: científico renacentista del siglo XX visto desde la cardiología del siglo XXI. En: Ramos Lara MP, Mansilla Corona RL, Mendoza Rosas RM, editores. Arturo Rosenblueth. Legado y vigencia de sus contribuciones. Ciudad de México: Universidad Nacional Autónoma de México, Centro de Investigaciones Interdisciplinarias en Ciencias y Humanidades; 2019. 259-74.

6. Bartlett RH, Gazzaniga AB. Extracorporeal circulation for cardiopulmonary failure. Curr Probl Surg. 1978;15:1-96.

7. Boink GJ, Christoffels VM, Robinson RB, Tan HL. The past, present, and future of pacemaker therapies. Trends Cardiovasc Med. 2015;25:661-73.

8. Beaupré RA, Frazier $\mathrm{OH}$, Morgan JA. Total artificial heart implantation as a bridge to transplantation: a viable model for the future? Expert Rev Med Devices. 2018;15:701-6.

9. Copeland JG, Smith RG, Arabia FA, Nolan PE, McClellan D, Tsau PH, et al. Total artificial heart bridge to transplantation: a 9-year experience with 62 patients. J Heart Lung Transplant. 2004;23:823-31.

10. Morin E. Introducción al pensamiento complejo. Barcelona, España: Gedisa; 2011. p. 135.

11. Gabryśa $E$, Rybaczuka M, Kędziab A. Fractal models of circulatory system. Symmetrical and asymmetrical approach comparison. Chaos, Solitons \& Fractals 2005;24:707-15.

12. Braun E. Caos, fractales y cosas raras. México D.F.: Fondo de Cultura Económica; 1996. (Consultado el 30 de abril de 2016.) Disponible en: http://bibliotecadigital.ilce.edu.mx/sites/ciencia/volumen3/ciencia3/150/ $\mathrm{htm} / \mathrm{sec} \_20 . \mathrm{htm}$.

13. Récamier V, Izeddin I, Bosanac L, Dahan M, Proux F, Darzacq X. Single cell correlation fractal dimension of chromatin $A$ framework to interpret 3D single molecule super-resolution. Nucleus. 2014;5:75-84.

14. Mancardi D, Varetto G, Bucci E, Maniero F, Guiot C. Fractal parameters and vascular networks: facts \& artifacts. Theor Biol Med Model. 2008:5:12.

15. Fossan FE, Mariscal-Harana J, Alastruey J, Hellevik LR. Optimization of topological complexity for one-dimensional arterial blood flow models. J R Soc Interface. 2018;15:20180546.

16. Gopinath B, Wang SB, Liew G, Phan K, Joachim N, Burlutsky G, et al. Retinal vascular geometry and the prevalence of atrial fibrillation and heart failure in a clinic-based sample. Heart Lung Circ. 2019;28:1631-7.

17. Nayak SK, Bit A, Dey A, Mohapatra B, Pal K. A review on the nonlinear dynamical system analysis of electrocardiogram signal. J Healthc Eng. 2018;2018:6920420.

18. Jovic A, Bogunovic N. Electrocardiogram analysis using a combination of statistical, geometric, and nonlinear heart rate variability features. Artif Intell Med. 2011;51:175-86.

19. Baillie RT, Cecen AA, Erkal C. Normal heartbeat series are nonchaotic, nonlinear, and multifractal: new evidence from semiparametric and parametric tests. Chaos. 2009;19:028503.

20. Karavaev AS, Ishbulatov YM, Ponomarenko VI, Bezruchko BP, Kiselev AR, Prokhorov MD. Autonomic control is a source of dynamical chaos in the cardiovascular system. Chaos. 2019;29:121101.

21. Babloyantz A, Destexhe A. Is the normal heart a periodic oscillator? Biol Cybern. 1988;58:203-11.

22. Bolea J, Laguna P, Remartínez JM, Rovira E, Navarro A, Bailón R Methodological framework for estimating the correlation dimension in HRV signals. Comput Math Methods Med. 2014;2014:129248.

23. Herbert-Read JE. Understanding how animal groups achieve coordinated movement. J Exp Biol. 2016;219(Pt 19):2971-83.

24. Babloyantz A. Mechanisms of target and spiral wave propagation in single cells. Chaos. 1994;4:473-6.

25. Majumder R, Nayak AR, Pandit R. Nonequilibrium arrhythmic states and transitions in a mathematical model for diffuse fibrosis in human cardiac tissue. PLoS One. 2012;7:e45040.

26. ten Tusscher KH, Noble D, Noble PJ, Panfilov AV. A model for human ventricular tissue. Am J Physiol Heart Circ Physiol. 2004;286:H1573-89.

27. Cherry $E$ and Fenton $F$. The virtual heart. http://thevirtualheart.org

28. Bartocci E, Cherry EM, Glimm J, Grosu R, Smolka SA, Fenton FH. Toward real-time simulation of cardiac dynamics, CMSB 2011 9th International Conference on Computational Methods in Systems Biology, Sept. 21-23 pages 103-110. ACM, 2011. (Consultado el 10 de junio de 2016.) Disponible en: http://thevirtualheart.org/FentonCherry/pdf/gpu.pdf.

29. Garfinkel A, Chen PS, Walter DO, Karagueuzian HS, Kogan B, Evans SJ, et al. Quasiperiodicity and chaos in cardiac fibrillation. J Clin Invest. 1997;99:305-14 
30. Weiss JN, Garfinkel A, Karagueuzian HS, Qu Z, Chen PS. Chaos and the transition to ventricular fibrillation: a new approach to antiarrhythmic drug evaluation. Circulation. 1999;99:2819-26.

31. Majumder R, Nayak AR, Pandit R. Scroll-wave dynamics in human cardiac tissue: lessons from a mathematical model with inhomogeneities and fiber architecture. PLoS One. 2011;6:e18052.

32. Brindle RC, Ginty AT, Phillips AC, Fisher JP, McIntyre D, Carroll D. Heart rate complexity: a novel approach to assessing cardiac stress reactivity. Psychophysiology. 2016;53:465-72

33. Delliaux S, Delaforge A, Deharo JC, Chaumet G. Mental workload alters heart rate variability, lowering non-linear dynamics. Front Physiol. 2019;10:565.

34. Ishiwata S, Shimamoto Y, Suzuki M. Molecular motors as an auto-osciIlator. HFSP J. 2010;4:100-4

35. Stoltz JF. History and future of hemorheology: from Reykjavik to Lisboa Clin Hemorheol Microcirc. 2016;64:525-39.

36. Boxenbaum $\mathrm{H}$. Interspecies scaling, allometry, physiological time, and the ground plan of pharmacokinetics. J Pharmacokinet Biopharm. 1982;10:201-27.

37. Craven BA, Aycock KI, Herbertson LH, Malinauskas RA. A CFD-based Kriging surrogate modeling approach for predicting device-specific hemolysis power law coefficients in blood-contacting medical devices. Biomech Model Mechanobiol. 2019;18:1005-30.

38. Lei H, Fedosov DA, Caswell B, Karniadakis GE. Blood flow in small tubes: quantifying the transition to the non-continuum regime. J Fluid Mech 2013;722.

39. Alarcon T, Byrne HM, Maini PK. A design principle for vascular beds: the effects of complex blood rheology. Microvasc Res. 2005;69:156-72.

40. Clabby C. Blood in motion. Applied mathematician George Karniadakis models how diseases alter the body's circulation. American Scientist. 2013;101:386-9.
41. De Brouwer S, Edwards DH, Griffith TM. Simplification of the quasiperiodic route to chaos in agonist-induced vasomotion by iterative circle maps. Am J Physiol. 1998;274:H1315-26.

42. Schmidt JA, Intaglietta M, Borgstrom P. Periodic hemodynamics in skeletal muscle during local arterial pressure reduction. J Appl Physiol. 1992;73:1077-83.

43. Parthimos D, Edwards DH, Griffith TM. Comparison of chaotic and sinusoidal vasomotion in the regulation of microvascular flow. Cardiovasc Res. 1996;31:388-99.

44. Zheng Y, Guo X. Identification of chronic heart failure using linear and nonlinear analysis of heart sound. Conf Proc IEEE Eng Med Biol Soc. 2017;2017:4586-9.

45. Tavares BS, de Paula Vidigal G, Garner DM, Raimundo RD, de Abreu LC, Valenti VE. Effects of guided breath exercise on complex behaviour of heart rate dynamics. Clin Physiol Funct Imaging. 2017;37:622-9.

46. Ilarraza H, Rodríguez L, Salas A, Ilarraza A, Rius M, Barrera C, et al. Análisis del acoplamiento corazón-pulmón mediante el espectro de potencias. XXVIII Congreso Mexicano de Cardiología, VII Congreso lberoamericano de Rehabilitación Cardiaca y Prevención Secundaria y 1 . $^{\text {er }}$ Congreso Mexicano de Rehabilitación Cardiovascular, Prevención y Cardiología del Deporte. Arch Cardiol Mex. 2013;83(Supl 3):19.

47. Mangin L, Clerici C, Similowski T, Poon CS. Chaotic dynamics of cardioventilatory coupling in humans: effects of ventilatory modes. Am J Physiol Regul Integr Comp Physiol. 2009;296:R1088-97.

48. Acharya UR, Chua EC, Faust O, Lim TC, Lim LF. Automated detection of sleep apnea from electrocardiogram signals using nonlinear parameters. Physiol Meas. 2011;32:287-303.

49. Ilarraza-Lomelí H. A cataclysm in the myocardium. Arch Cardiol Mex. 2017;87:151-4. 